\title{
Progressive pseudorheumatoid arthropathy of childhood
}

INSERM

\section{Source}

INSERM. (1999). Orphanet: an online rare disease and orphan drug data base.

Progressive pseudorheumatoid arthropathy of childhood. ORPHA:1159

Progressive pseudorheumatoid arthropathy (dysplasia) of childhood (PPAC; PPD)

presents as spondyloepiphyseal dysplasia (SED) tarda with progressive arthropathy and is described as a specific autosomal recessive subtype of SED. 